\title{
Validating Machining Tools using Square Sum Labeling Technique
}

\author{
K. Manimegalai, G. Subashini
}

\begin{abstract}
This work claims that the SS labeling for generalized web without centreWO $(m, 3)$, corona graph (Pm $x$ C3) $\odot K 1$ for and (Pm $x$ C3).
\end{abstract}

Keywords: square sum labeling(SSL),SSA, WO (m,n), (Pm $x$ C3) $\odot$ Kland BFS Algorithm. AMS Classification No: $05 C 78$

\section{INTRODUCTION}

All graphs are simple, conditioned and irregular. For all terminologies follow as in [1]. A study of graphs which are square sum and strongly square sum by [4] was initiated. In short square sum labeling is denoted by SSL, for some middle and total graphs are proved in [10]. Magic labeling of Pm $\times C n$ for $m \geq 2$ and $W_{0}(m, n)$ for odd $n \geq 3$ graphs was proved by $[8,9]$.

Definition 1.1: consider a SS graph, if there exist a function which is both a surjection and an injection, such that it give rise to function $f^{*}$ mapping from an edges to the set of natural numbers given

by $f^{*}(u v)=[f(u)]^{2}+[f(v)]^{2}$ for every $u v \in E(G)$ is injective. A graph which admits SSL is called SS graph.

Example: SSL for $\mathrm{k}_{1,6}$ isshown in figure 1.1.

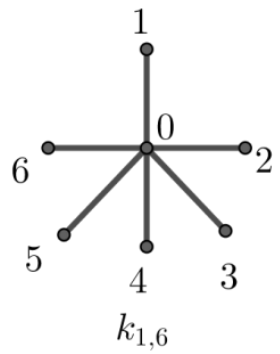

fig1.1 : square sum labeling

Definition 1.2: $W_{0}(m, n)$ is the graph with vertex set $V=\left\{v_{0}, v_{i, j} / 1 \leq i \leq m+1,1 \leq j \leq n\right\}$ and the edge set

$$
E=\left\{v_{i, j} v_{i, j+1}, v_{i, j} v_{i+1, j}, v_{0} v_{1, j} / 1 \leq i \leq m, 1 \leq j \leq n\right\}
$$

where $\mathrm{j}$ is taken modulo $\mathrm{n}$ (replacing 0 by $\mathrm{n}$ ). $W_{0}(m, n)$ and its illustration is seen in figure 1.2.

Revised Manuscript Received on December 16, 2019

K. Manimegalai, Associate Professor, Department of Science and Humanities, Bharath Institute of Higher Education and Research, India manimekalai2010@yahoo.com

G. Subashini, Assistant Professor, Department of Science and Humanities, Bharath Institute of Higher Education and Research, India, suba.thulam@gmail.com

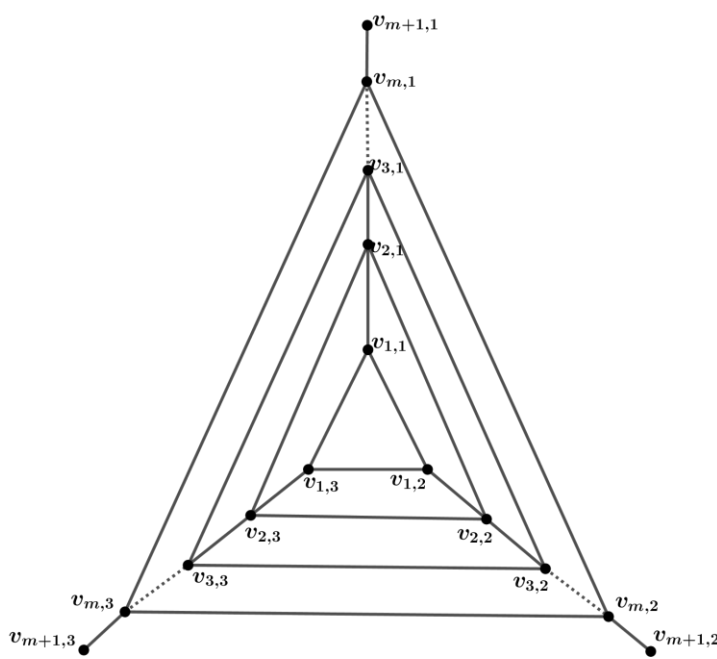

Figure 1.2

\section{Definition 1.3}

$\mathrm{TheP}_{\mathrm{m}} \times \mathrm{C}_{\mathrm{n}}$ is the graph called prism graph which is both planar and polyhedral and its examined below in figure 1.3

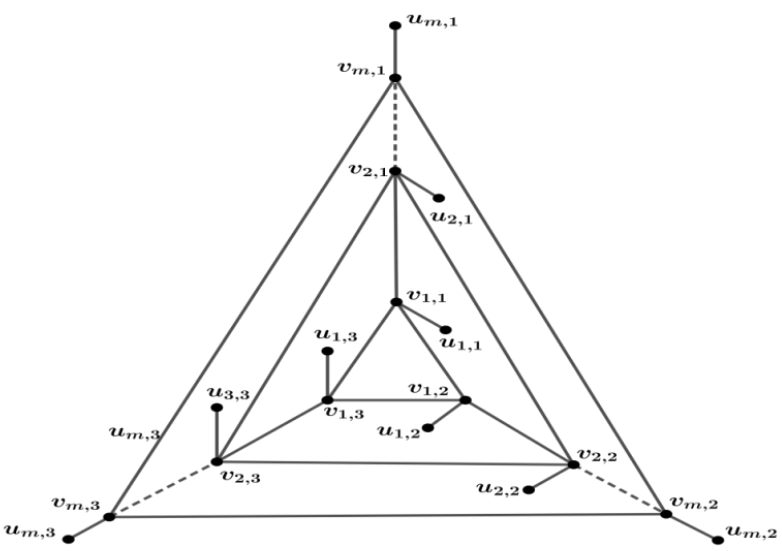

Figure 1.3

\section{MAIN RESULTS}

Theorem 2.1: Web without centre $W_{0}(m, 3)$ is a SS-graph for $n \geq 3, m \geq 2$

Proof: Let $\mathrm{G}$ be the web without centre graph with a vertex $\left|V^{\prime}\right|=(m+1) n$ and the edge $\left|E^{\prime}\right|=6 m$. Define a one to one and onto function $f: V^{\prime} \rightarrow\{0,1,2, \ldots, 3(m+1)-1\}$ as follows: choose a vertex $v_{m+1,1}$ as a root vertex and label it as 0 and visit remaining vertices by using BFS algorithm pattern in anticlockwise direction or labeling from left to right with consecutive numbers $1,2,3, \ldots, 3(m+1)$-1 rises in order, in the way in which they are visited in a one to one manner. 
Clearly finduces a map $f^{*}: E^{i} \rightarrow N$ as defined by $f^{*}(x y)=\left[f(x)^{2}\right]+\left[f(y)^{2}\right]$ for every $\quad w \in E^{\prime}$. Using the induced function, we classify $E^{\prime}$ into two sets as follows:

The set of edges with both of their end vertices have labels either odd or even integers or each edge has end vertices with one end vertex has odd integer and the other has even integer as its labels. Then the sum squares of the labels of end vertices of each edge is an odd and even numbers respectively, and these numbers form a strictly increasing sequence of even integers.

Hence it is observed that $f^{*}\left(e_{i}\right) \neq f^{*}\left(e_{j}\right)$.So, it is obvious that from the above defined the procured function $f^{*}$ is injective Hence $W_{0}(m, 3)$ is a SSG.

Example 2.2: The $W_{0}(4,3)$ is square sum graph shown in figure 2.1.

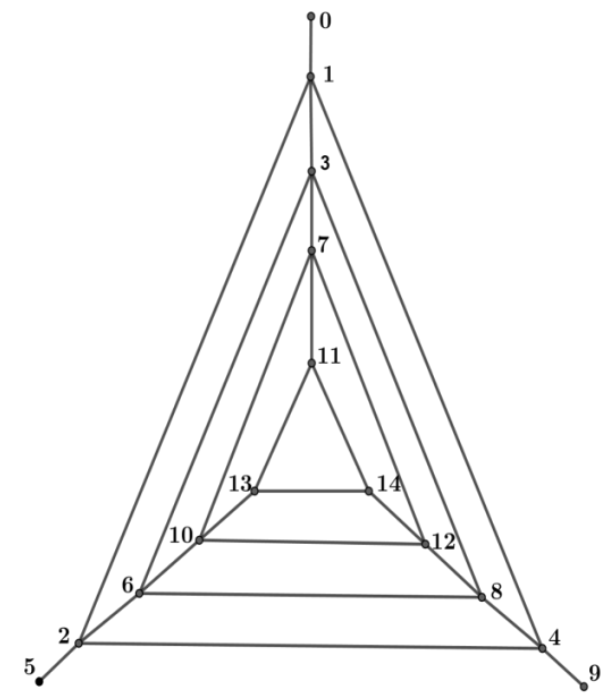

Figure 2.1

\section{Theorem 2.2}

For $\mathrm{m} \geq 2$, the corona graph $\left(P_{m} \mathrm{x} C_{3}\right) \odot \mathrm{K}_{1}$ admits SSL.

\section{Proof}

Consider a graph $\mathrm{G}=\left(P_{m} \mathrm{x} C_{3}\right) \odot K_{1}$ with cardinality of vertex $6 m$ and edges $3(3 m-1)$.

Define the vertex labelingfmapping from vertex to $\{0,1,2, \ldots, 6 m-1\}$ as given below:

$\mathrm{xChoose}$ any pendent vertex attached with the outer most cycle, starting from the vertex visit all the vertices of $\mathrm{G}$ using $\mathrm{BFSalgorithm}$ and label the vertices. Hence $\mathrm{f}$ is bijective. The induced function are classified into two classes as in the proof

Clearly it is seen that the induced function $f^{*}$ is injective. Hence the corona graph $\left(P_{m} \mathrm{x} C_{3}\right) \odot \mathrm{K}_{1}$, for $m \geq 2$ attains SSL.

\section{Example 2.2}

The SSL of corona graph $\left(\mathrm{P}_{3} \times \mathrm{C}_{3}\right) \odot \mathrm{K}_{1}$ for $m \geq 2$ is shown in figure2.2. of theorem 2.1.

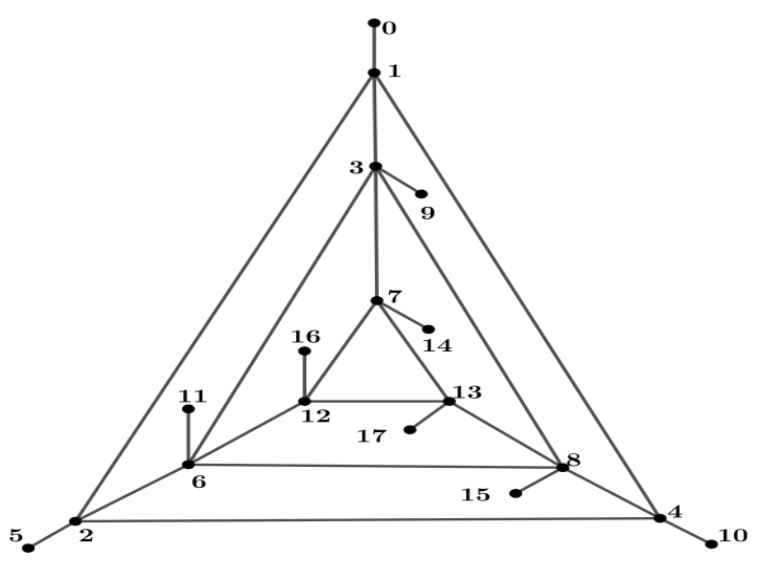

Figure 2.2

\section{Theorem 2.3}

For $m \geq 2, P_{m} \times C_{\mathrm{a}}$ admits QDL.

Proof:Consider $P_{m} \times C_{a}$ be a graph with cardinality of vertices and edges, $3 m$ and $3(2 m-1)$ respectively.

Choose a vertex say $v_{1,1}$ as a root vertex and label it as 0 and visit remaining vertices by using BFS algorithm pattern in anticlockwise direction or label the vertices from left to right with the consecutive numbers $1,2,3, \ldots,(3 m-1)$ in ascending order.

By Mani's lemma[8], If $0<a<b$, then $a^{2}+b^{2}<(a-c)^{2}+(b+c)^{2}$ for $1 \leq c \leq a$ and $a, b, c \in N$, its clearly observed that the labels of the edges form an increasing sequence and hence the induced edge labelings are injective and are distinct. Hence $P_{m} \times C_{\mathrm{a}}$ is a SSG.

\section{Example 2.3}

A graph $P_{a} \times C_{a}$ is illustrated below in figure 2.3.

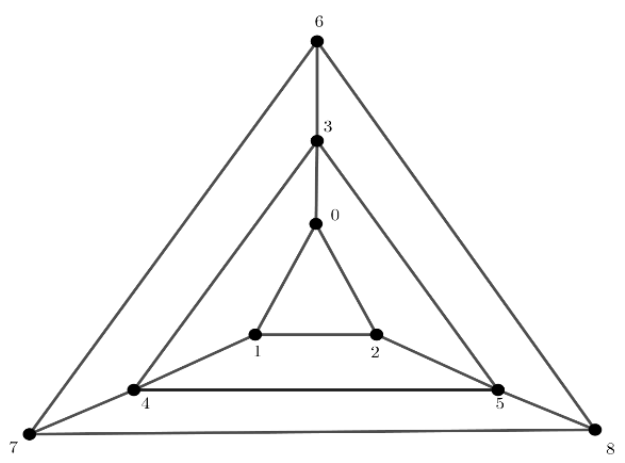

Figure 2.3

\section{CONCLUSION}

Here we've proved the result on SSL of $W_{0}(m, n)$, corona graph $\left(\mathrm{P}_{\mathrm{m}} \times \mathrm{C}_{3}\right) \odot \mathrm{K}_{1}$ for $m \geq 2$ and $\mathrm{P}_{\mathrm{m}} \times \mathrm{C}_{3}$.

\section{REFERENCE}

1. Ajitha.V, S.Arumugam and K.A.Germina, "On square sum graphs", AKCE J. Graphs and combinatorics, 6(1), (2009), 1-10

2. Beineke.L.W. And S.M.Hegde, Discuss. Math. Graph Theory 21, 63 (2001)

3. Gallian, J.A. "A dynamic survey of graph labeling” The Electronic Journal of Combinatorics (2017), \#DS6.

4. Germina.K.A and R.Sebastian "Further results on square sum

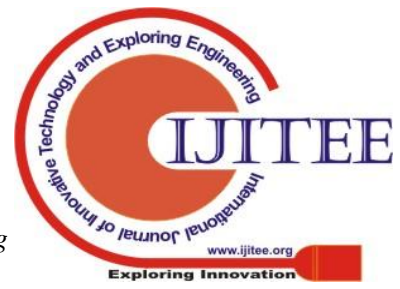


graphs" International Mathematical forum, Vol. 8, 2013, no. 1, 47-57

5. F.Harary. "Graph theory" Narosa Publishing House (2001).

6. Figuerora-Centeno R.M., Ichisima R., Muntaner-Batle F.A., 2001. The place of super edge- magic labeling among other classes of labeling, Discrete Math. 231, 153-168.

7. Lee.S.M and G. Wang, All pyramids, lotuses and diamonds are k -graceful, Bull. Math. Soc. Sci. Math. R. S. Roumanie (N. S), 32(1988) 145-150.

8. K. Manimekalai, S. Manisha and P. Jagadeeswari, On Square Sum Labeling Of Cycle Related Graphs, Advancement in Engineering, Science \& Technology J. Mech. Cont.\& Math. Sci., Special Issue, No.-2, August (2019) pp 618-626.

9. Manimekalai. K and K.Thirusangu, Super Edge-magic and Super Edge-bimagic Total labeling of some special graphs, Proceedings of International Conference On Mathematical Computer Engineering, Vol.III, (ICMCE-2013), VIT Chennai, (2013) 965-972.

10. Manimekalai. K and K. Thirusangu, Antimagic, Super Edge-Magic And Edge-Bimagic Labelings Of Some Graphs. International Journal of Pure and Applied Mathematics Volume 101 No. 6 2015, 1041-1050.

11. Rosa. A, on certain valuation of graph, theory of graphs (Rome, July 1966), Golden and Breach. N.Y and Paris, (1967), 349 - 355.

12. Shiama .J, "Square sum labeling for some middle and total graphs" International Journal of Computer Applications (0975-8887), Vol.37-No 4. January 2012.

\section{AUTHORS PROFILE}

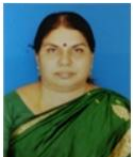

K. Manimegalai, Associate Professor, Department of Science and Humanities, Bharath Institute of Higher Education and Research, India

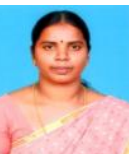

G. Subashini, Assistant Professor, Department of Science and Humanities, Bharath Institute of Higher Education and Research, India 